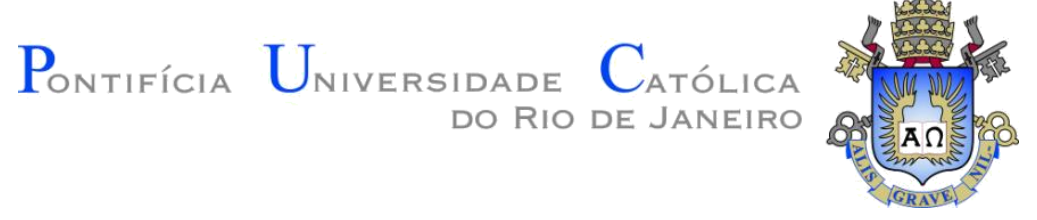

Fabián Martín Vizcarra Campana

Modelagem e Simulação do Transporte e
Remediação de Poluentes em Aqüíferos

Dissertação de Mestrado

Dissertação apresentada como requisito parcial para obtenção do grau de Mestre pelo Programa de Pós-Graduação em Engenharia Mecânica da PUC-Rio.

Orientador: Prof. Arthur Martins Barbosa Braga Co-Orientador: Eurípedes do Amaral Vargas Júnior 
Fabián Martín Vizcarra Campana

\section{Modelagem e Simulação do Transporte e Remediação de Poluentes em Aqüíferos}

Dissertação apresentada como requisito parcial para obtenção do grau de Mestre pelo Programa de Pós-Graduação em Engenharia Mecânica da PUC-Rio. Aprovada pela Comissão Examinadora abaixo assinada.

Prof. Arthur Martins Barbosa Braga

Orientador

Departamento de Engenharia Mecânica - PUC-Rio

Prof. Eurípedes do Amaral Vargas Jr. Co-Orientador Departamento de Engenharia Civil - PUC-Rio

Prof. Marcos Sebastião de Paula Gomes Departamento de Engenharia Mecânica - PUC-Rio

Profa. Andrea Ferreira Borges Departamento de Geologia - UFRJ

Prof. José Eugenio Leal Coordenador Setorial do Centro Técnico Científico - PUC Rio 
Todos os direitos reservados. E proibida a reprodução total ou parcial do trabalho sem a autorização da universidade, do autor e do orientador.

\section{Fabián Martín Vizcarra Campana}

Fabián Vizcarra é formado como Físico pela Universidad Nacional Mayor de San Marcos (UNMSM), Universidad del Perú, Decana de América, fundada el 12 de mayo de 1551. Seu trabalho de pesquisa é na linha da físicoquímica de fluidos em meios porosos geologicos e na física teórica.

Ficha Catalográfica

Vizcarra Campana, Fabián Martín

Modelagem e simulação do transporte e remediação de poluentes em aqüíferos / Fabián Martín Vizcarra Campana ; orientador: Arthur Martins Barbosa Braga ; co-orientador: Eurípedes do Amaral Vargas Júnior. - 2014.

97 f. : il. (color.) ; $30 \mathrm{~cm}$

Dissertação (mestrado)-Pontifícia Universidade Católica do Rio de Janeiro, Departamento de Engenharia Mecânica, 2014.

Inclui bibliografia

1. Engenharia mecânica - Teses. 2. Remediação de aquíferos. 3. Hidráulica dos solos. 4. Modelagem numérica de escoamento em meios porosos. I. Braga, Arthur Martins Barbosa. II. Vargas Júnior, Eurípedes do Amaral. III. Pontifícia Universidade Católica do Rio de Janeiro. Departamento de Engenharia Mecânica. IV. Título.

CDD: 621 


\section{Agradecimentos}

Primeiramente muito agradecido com a PUC-Rio pela bolsa de isenção do curso.

Ao CNPq pelo auxílio financeiro concedido durante o mestrado.

Ao Brasil, pais que me da oportunidade de desenvolver-me como Professional e pesquisador. Infinitamente agradecido.

Ao meu orientador e co-orientador, os professores Arthur Braga e Eurípedes Vargas. Um honor pra me trabalhar com eles. Obrigado por tudo seu apoio e conselhos para o desenvolvimento de esta dissertação. 


\section{Resumo}

Campana, Fabián Martín Vizcarra; Braga, Arthur Martins Barbosa. Modelagem e Simulação do Transporte e Remediação de Poluentes em Aquííferos. Rio de Janeiro, 2014. 97pp. Dissertação de Mestrado Departamento de Engenharia Mecânica, Pontifícia Universidade Católica do Rio de Janeiro.

Apresenta-se nesta dissertação um estudo dos principais mecanismos do modelo de fluxo subterrâneo e do modelo de transporte de poluentes em aqüíferos. Os processos associados ao transporte de poluentes são estudados em detalhe: difusão, dispersão e retenção de contaminantes na matriz porosa do aqüífero. Seguidamente é desenvolvido, com o apoio do software Visual MODFLOW v.2011.1, duas simulações numéricas com dados sintéticos do transporte e remediação de um poluente na zona saturada de um aqüífero composto por três camadas. O objetivo primeiro da simulação computacional do modelo de fluxo subterrâneo e do modelo de transporte de poluentes é avaliar os modelos teóricos estudados e fornecer uma guia metodológica da simulação computacional da dinâmica de aguas subterrâneas e da evolução das plumas poluentes sometidas a condiciones externas; como por exemplo: poços de bombeamento, poços de injeção, infiltrações, etc. Os dados empregados na simulação representam um aqüífero sintético composto por três camadas com uma falha geológica no meio do sistema, o que permite conectar hidraulicamente a camada superficial, representada por um aquíifero freático, com a camada representada por um aqüífero confinado. $\mathrm{O}$ segundo objetivo é simular numericamente o processo de remediação de aquíferos poluídos, com a finalidade de estabelecer a efetividade do método. Particularmente foi escolhido o método de remediação Pump-and-Treat, um dos sistemas de tratamento de aqüíferos mais utilizados para este fim, que consiste no bombeamento para a superfície das aguas subterrâneas poluídas para posterior tratamento externo de remoção de contaminantes.

\section{Palavras-chave}

Remediação de aquíferos; hidráulica dos solos; modelagem numérica de escoamento em meios porosos. 


\begin{abstract}
Campana, Fabián Martín Vizcarra; Braga, Arthur Martins Barbosa. Modeling and Simulation of Transport and Remediation of Poluants in Aquifers. Rio de Janeiro, 2014. 97pp. MSc. Dissertation - Departamento de Engenharia Mecânica, Pontifícia Universidade Católica do Rio de Janeiro.
\end{abstract}

We present in this paper a study of the main mechanisms of the model groundwater flow and transport model of pollutants in aquifers. The processes associated with the transport of pollutants are studied in detail: diffusion, dispersion and retention of contaminants in the aquifer porous matrix. Is then developed with the support of Visual MODFLOW software v.2011.1, two numerical simulations with synthetic data transport and remediation of a pollutant in the saturated zone of an aquifer composed of three layers. The primary goal of numerical simulation of the groundwater flow model and transport of pollutants studied model is to evaluate the theoretical models and provide a methodological guide computer simulations of the dynamics of ground water and the evolution of pollutant plumes sometidas external condiciones; for example: pumping wells, injection wells, infiltration, etc.. The data used in the simulation represents a synthetic aquifer consists of three layers with a fault in the middle of the system, which allows for hydraulically connecting the surface layer represented by a water aquifer, with the layer represented by a confined aquifer. The second goal is to numerically simulate the process of remediation of polluted aquifers, in order to establish the effectiveness of the method. Particularly was chosen remediation method Pump-and-Treat, treatment of the aquifer most systems used for this purpose, which consists of pumping to the surface of the polluted groundwater for further treatment to remove external contaminants.

\title{
Keywords
}

Aquifer remediation; soil hydraulics; numerical simulation of flow in porous media. 


\section{Sumario}

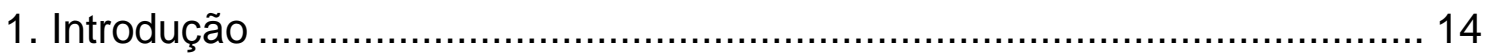

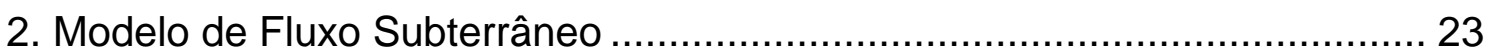

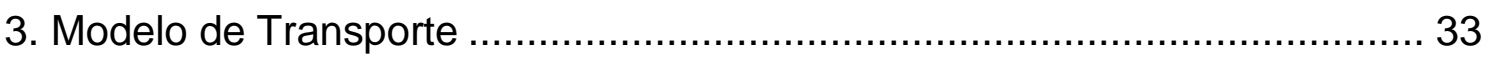

4. Simulação Numérica do Fluxo e Transporte ……………........................... 47

5. Simulação Numérica da Técnica Pump-and-Treat ................................... 78

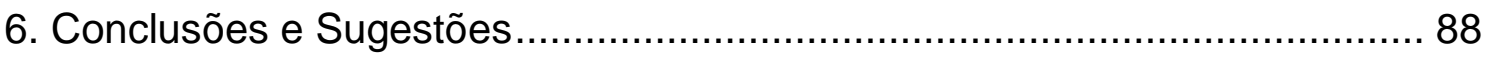

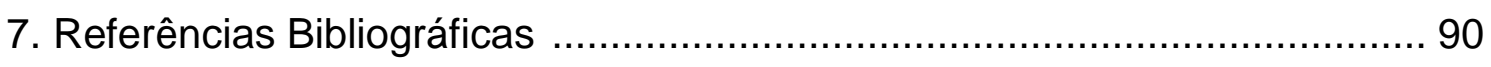

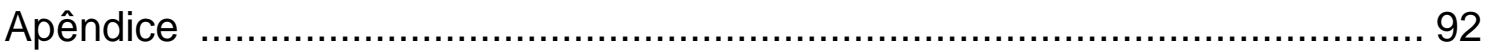




\section{Lista de Figuras}

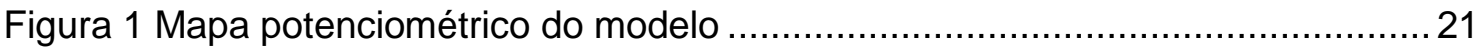

Figura 2 Mapa de concentração de poluentes do modelo MT3DMS ...........................22

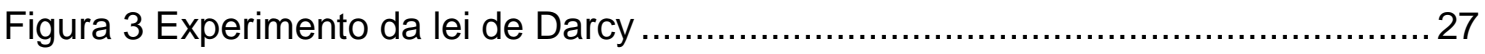

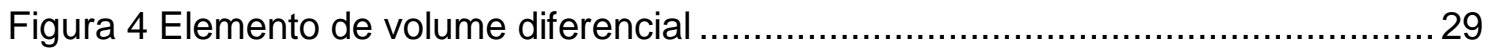

Figura 5 Efeito dos processos do transporte para uma fonte continua ........................39

Figura 6 Efeito dos processos do transporte para uma fonte de tipo pontual ..............40

Figura 7 Expressão logarítmica de Freundlich (Alfaro, 2005) ................................... 44

Figura 8 Modelo linear de sorção (Alfaro, 2005) …................................................ 44

Figura 9 Efeito da biodegradação para uma fonte do poluente continua .................... 45

Figura 10 Efeito da biodegradação para uma fonte do poluente de tipo pontual ..........46

Figura 11 Plano representativo do modelo conceitual...............................................52

Figura 12 Distribuição e tipos de aquíferos do modelo do reservatório ........................52

Figura 13 Malha do modelo da zona de estudo ................................................... 54

Figura 14 Pontos das mostras de campo para a extrapolação...................................56

Figura 15 Interpolação da estratigrafia real do modelo .........................................57

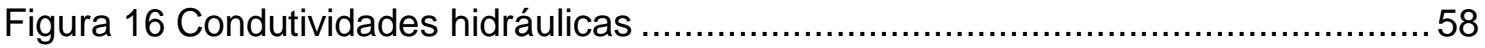

Figura 17 Escala das cargas hidráulicas em metros ............................................ 59

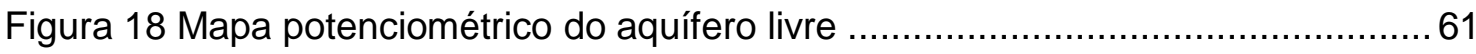

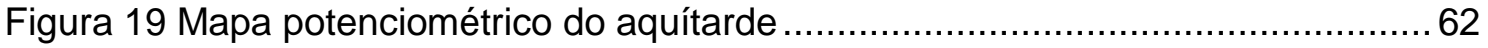

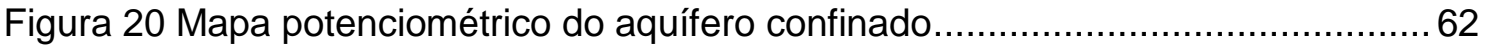

Figura 21 Mapa potenciométrico na zona da descontinuidade geológica.................... 63

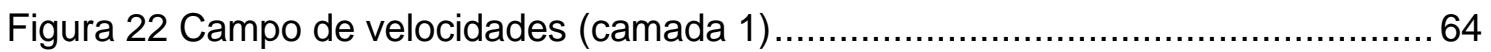

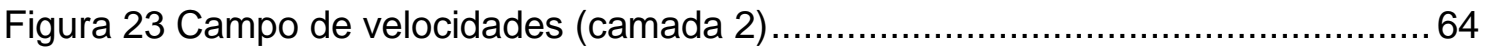


Figura 24 Campo de velocidades (camada 3).....

Figura 25 Campo de velocidades no modelo bidimensional vertical na zona

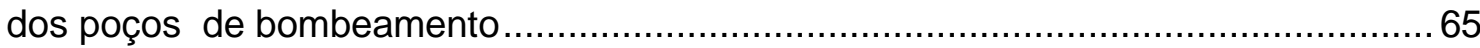

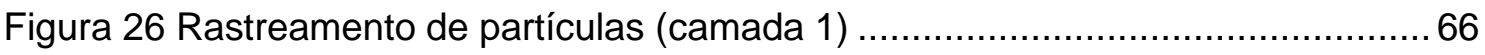

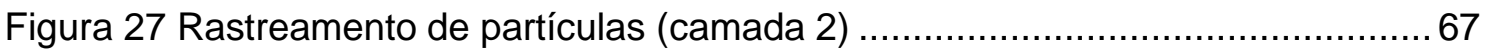

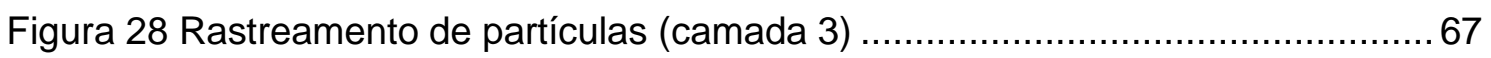

Figura 29 Rastreamento de partículas (Modelo bidimensional vertical) ......................68

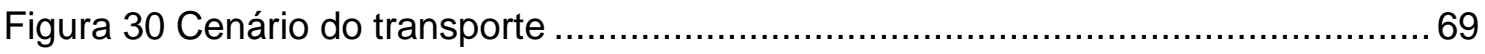

Figura 31 Escala de concentração de poluentes............................................... 69

Figura 32 Dados da concentração do poluente fornecidos pelos ............................... 72

Figura 33 Pluma contaminante no aquifero livre (730 dias).................................. 73

Figura 34 Pluma contaminante no aquífero livre (1460 dias) …............................. 74

Figura 35 Pluma contaminante no aquífero livre (7300 dias) ................................... 74

Figura 36 Modelo bidimensional vertical da pluma contaminante ............................. 75

Figura 37 Pluma contaminante no aquitardo (7300 dias) …................................... 75

Figura 38 Evolução espaciotemporal da pluma poluente, do tempo T2 ate

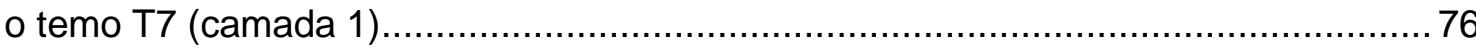

Figura 39 Evolução espaciotemporal da pluma poluente, do tempo T2 ate

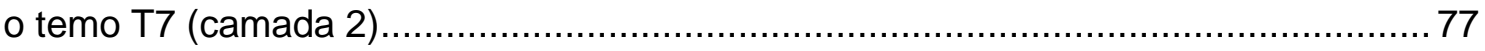

Figura 40 Evolução espaciotemporal da pluma poluente, do tempo T2 ate o tempo T7

Figura 41 Evolução temporal da pluma poluente, do tempo T4 ate o tempo T7

Figura 42 Modelo de tratamento Pump-and-Treat fundamentado na interceptação hidráulica da pluma poluente

Figura 43 Caraterísticas operacionais dos poços

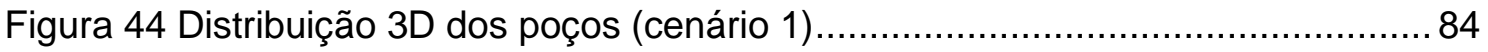

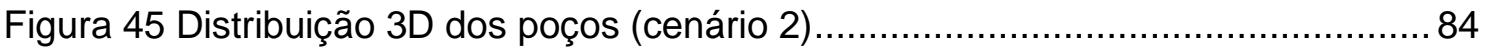

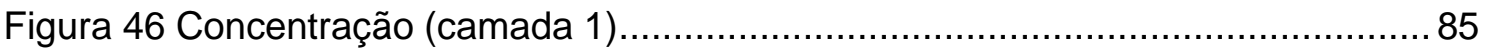

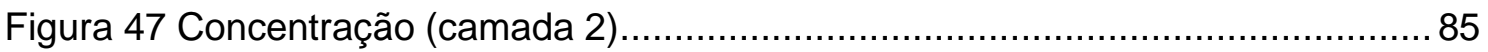




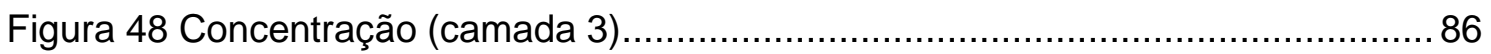

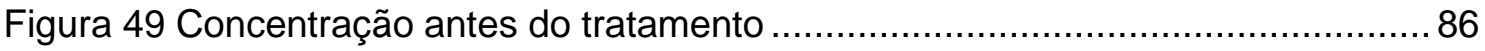

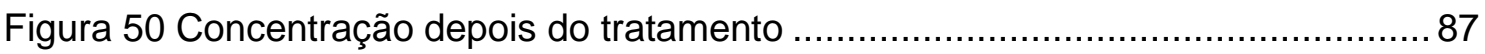

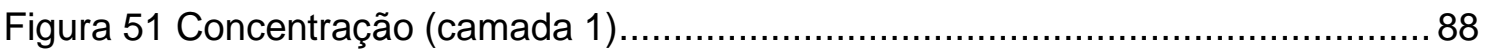

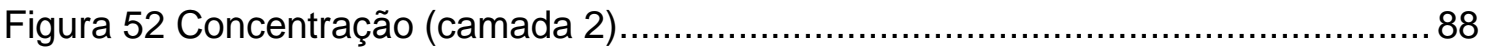

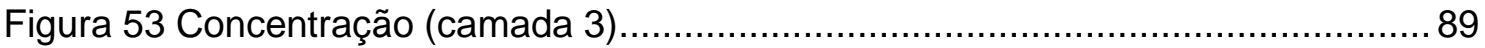

Figura 54 Concentração depois do tratamento (cenário 2) ..................................... 89

Figura 55 Volume de controle com dimensões Dx x Dy x Dz ....................................94 


\section{Lista de Tabelas}

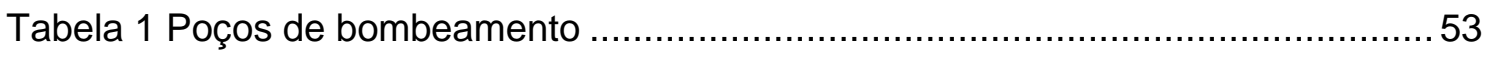

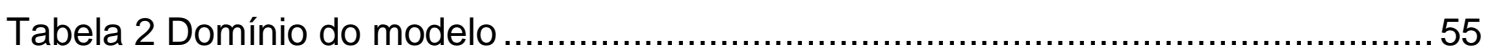

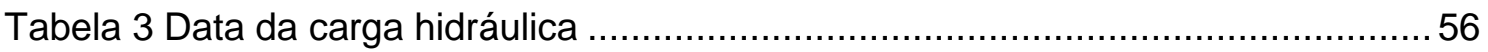

Tabela 4 Condutividade hidráulica das camadas .................................................. 57 


\section{Lista de Símbolos}

A - Área da secção transversal $\left[\mathrm{L}^{2}\right]$

$b$ - espessura do aquífero [L]

$C_{s}$ - Concentração da fonte $\left[\mathrm{ML}^{-3}\right]$.

C- Concentração do soluto dissolvido na água subterrânea $\left[\mathrm{ML}^{-3}\right]$.

$C^{*}$ - Massa de poluente adsorvido por unidade de peso da fase sólida [\%].

$D^{*}$ - Coeficiente de difusão em meios porosos $\left[\mathrm{L}^{2} \mathrm{~T}^{-1}\right]$.

$D$ - Coeficiente de difusão em água livre $\left[\mathrm{L}^{2} \mathrm{~T}^{-1}\right]$.

$D_{i j}$ - Tensor de dispersão hidrodinâmica $\left[\mathrm{L}^{2} \mathrm{~T}^{-1}\right]$.

$D_{m x}$ - Coeficiente de macrodispersão no sentido do fluxo subterrâneo $\left[\mathrm{L}^{2} \mathrm{~T}^{-1}\right]$.

$F$ - volume de fluido por diferencial de tempo e por unidade de volume $\left[\mathrm{ML}^{-2} \mathrm{~T}^{-1}\right]$.

$g$ - Aceleração da gravidade $\left[\mathrm{LT}^{-2}\right]$

$q_{s}$-Taxa de fluxo de água por unidade de área normal à direção do fluxo [ $\left[T^{-1}\right]$.

$q$ - Velocidade de Darcy [ $\left[\mathrm{LT}^{-1}\right]$

$Q$ - Vazão do fluxo subterrâneo $\left[\mathrm{L}^{3} \mathrm{~T}^{-1}\right]$

$K$ - Condutividade hidráulica ou permeabilidade $\left[\mathrm{LT}^{-1}\right]$.

$k$ - Permeabilidade intrínseca do meio poroso $\left[\mathrm{L}^{2}\right]$.

$K_{d}$ - Coeficiente de distribuição [-]

$n$ - Porosidade [\%]

$r$ - Termo de efeitos de adsorção e reação química $\left[\mathrm{ML}^{-3} \mathrm{~T}^{-1}\right]$.

$T^{*}$ - Tortuosidade [-].

$T$ - Transmissividade $\left[\mathrm{L}^{2} \mathrm{~T}^{-1}\right]$,

$S_{0}$ - Coeficiente de armazenamento específico $\left[\mathrm{L}^{-1}\right]$,

$v_{i}$ - Velocidade de transporte na direção $i\left[\mathrm{LT}^{-1}\right]$.

$V_{s}$-Volume da parte sólida $\left[\mathrm{L}^{3}\right]$.

$V_{v}$-Volume de vacíos $\left[\mathrm{L}^{3}\right]$.

$V_{t}$-Volume total $\left[\mathrm{L}^{3}\right]$.

$z$ é a altura da água [L]. 
$\theta$ - Porosidade efetiva ou eficaz [\%].

$\rho$ - Massa específica $\left[\mathrm{ML}^{-3}\right]$.

$\phi$ - Potencial hidráulico [L].

$\gamma$ é o peso específico $\left[\mathrm{ML}^{-3}\right]$.

$\alpha$ - Dispersividade $\left[\mathrm{L}^{2} \mathrm{~T}^{-1}\right]$.

$i$ - Gradiente hidráulica [-].

$\rho_{a}$ - Densidade aparente do solo $\left[\mathrm{ML}^{-3}\right]$.

$\sigma$ - Razão entre o volume de água contida no solo e volume da matriz porosa $[-]$.

$r_{f}$ - Fator de retardamento [-]. 